\title{
Acquisition of a microscope for in situ studies of hard and soft matter
}

\author{
James M. LeBeau ${ }^{1}$, Elizabeth C. Dickey ${ }^{1}$, Veronica Augustyn ${ }^{1}$, Dean L. Hesterberg ${ }^{2}$, Ashley C. Brown ${ }^{3}$ \\ 1. Department of Materials Science \& Engineering, North Carolina State University, Raleigh, USA \\ 2. Department of Crop and Soil Sciences, North Carolina State University, Raleigh, USA \\ 3. Department of Biomedical Engineering, North Carolina State University, Raleigh, USA
}

As interdisciplinary research areas involving carbon-based materials, energy materials, environmental science, and data science continue to rapidly grow, electron microscopes are required that can investigate hard and soft matter at conditions where relevant phenomena occur, i.e. other than at room temperature and in vacuum. Such areas of interest include: energy storage and conversion, biological compatibility, geochemical interactions, and exotic properties in oxide materials. To address research needs in these areas, the new instrument will catalyze research by enabling fast in situ imaging of dynamic material behavior in a variety conditions including low temperatures (down to cryogenic), heating, biasing, mechanical loading, gas, and liquid. In combination with the capability to capture three-dimensional data, this instrument will accelerate intellectual output by empowering researchers to make new, atomic scale insights, with greatly improved temporal resolution, and at conditions relevant to the scientific questions.

In this presentation, we will discuss the acquisition of a FEI Talos G2 transmission electron microscope (TEM) at North Carolina State University (NCSU), funded through the NSF MRI program. The instrument is equipped with a set of capabilities to answer scientific questions regarding the nanoscale structure of soft and hard matter. With the broad spectrum of materials and systems that can be studied with the new instrument, the acquisition impacts research across interdisciplinary areas. We will also point to the need for key preliminary results in writing proposals for new instruments. As shown in Figure 1, for example, preliminary data to should show how the instrument can answer key scientific questions from current user challenges [1].

In terms of accessibility, we will also discuss the need for a clear management plan. At NCSU, the microscopy will be managed by the Analytical Instrumentation Facility, which is the lead organization of the NSF NNCI Research Triangle Nanotechnology Network (RTNN) and brings $\sim 30$ years of experience in electron microscopy training, teaching, and maintenance. This open access center serves local users, providing training and hands-on usage of the instruments, as well as researchers across the nation through a mail-in program. While there is already an active and productive user base for hard matter microscopy at NCSU, the instrument brings critical soft matter TEM capabilities to shared user facilities at NCSU. To accelerate building the soft matter TEM community, we will proactively connect with RTNN users through short courses, workshops, and classroom integration. For outreach, the TEM will be integrated into several activities at department and facility levels, where K-12 students can experience scientific instrumentation first hand. Outreach is also key, and as such a key educational outreach program has been integrated, namely LeBeau's 'Atomic Resolution Electron Microscopy as Art Exhibit' that targets young students and the public by stimulating interest in science through the visual arts by integrating art and microscopy at the atomic scale [2]. 
References:

[1] J Mitchell, et al., Chemistry of Materials 29 (2017), p. 3928-3937.

[2] The author acknowledges funding from the National Science Foundation, Grant DMR-1726294 and DMR-1350273.
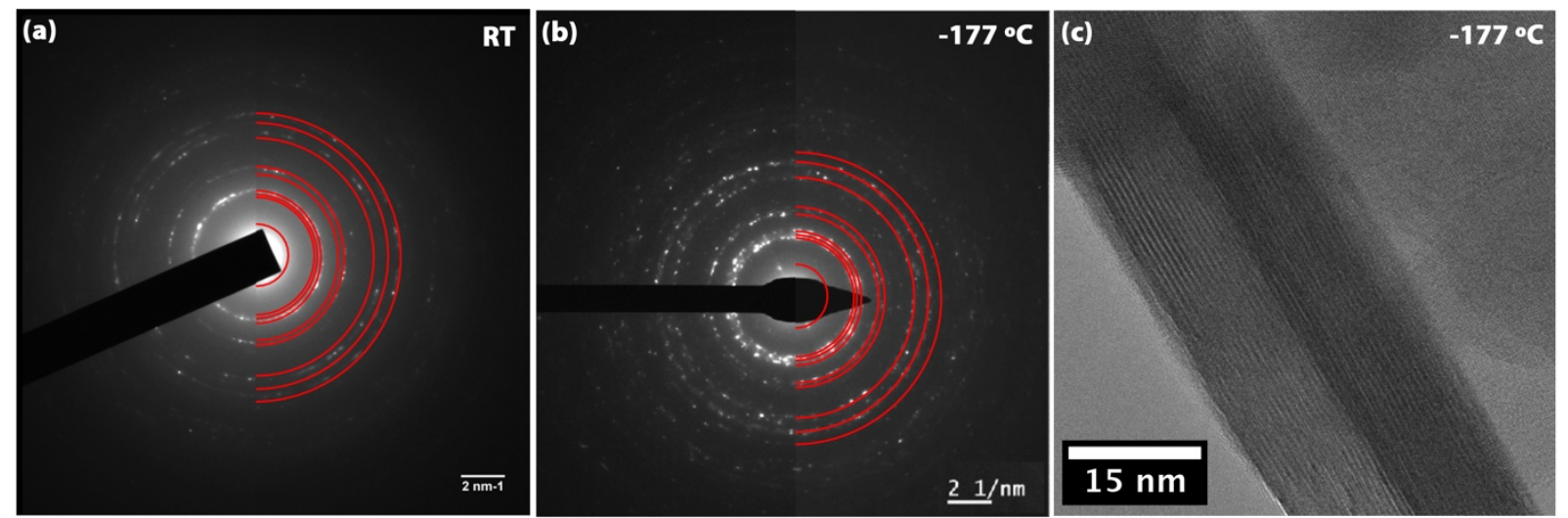

Figure 1. (a) Using previously available instruments at NCSU, the expected structure of $\mathrm{WO}_{3}-2 \mathrm{H}_{2} \mathrm{O}$ hydrated material is not observed at RT. Rings are from JCPDS file for $\mathrm{WO}_{3}-\mathbf{2} \mathrm{H}_{2} \mathrm{O}$. (b) With the Talos TEM at cryo conditions, the diffraction pattern closely matches the expected hydrated crystal structure. 